Pesq. Vet. Bras. 35(Supl.1):51-55, dezembro 2015 DOI: $10.1590 / \mathrm{S} 0100-736 \mathrm{X} 2015001300009$

\title{
Concentrações bioquímicas séricas de jacaré-açú (Melanosuchus niger) machos, adultos de vida livre ${ }^{1}$
}

\author{
Bruno Tolentino Caixeta ${ }^{2 *}$, Eva Mendes Monteiro², Paulo Vinícius Pereira Rocha ${ }^{2}$ \\ e André Luiz Quagliatto Santos ${ }^{2}$
}

\begin{abstract}
Caixeta B.T., Monteiro E.M., Rocha P.V.P. \& Santos A.L.Q. 2015. [Biochemical serum concentrations of adult male caimans (Melanosuchus niger) in free life.] Concentrações bioquímicas séricas de jacaré-açú (Melanosuchus niger) machos, adultos de vida livre. Pesquisa Veterinária Brasileira 35(Supl.1):51-55. Laboratório de Ensino e Pesquisa em Animais Silvestres, Universidade Federal de Uberlândia, Rua Ceará s/n, Bloco 2D, Sala 36, Bairro Umuarama, Uberlândia, MG 38405-315, Brazil. E-mail: brcaixeta@yahoo.com.br

The black caiman (Melanosuchus niger) of the Aligatoridae family is considered to be the largest aquatic predator of Continental America. Like all reptiles, the black caiman has special features for energy use to control his body temperature according to climatic variations. These variations can reveal the current metabolic status of the animals, explaining the importance to study such parameters. We determined the biochemical profile of some blood compounds of 12 alligators, weighing $77.66 \pm 8.92 \mathrm{~kg}$, as there are: urea $(3.41 \pm 0.66)$, albumin $(24.85 \pm 0.63)$, total protein $(63.83 \pm 16.97)$, uric acid $(0.25 \pm 0.09)$, glucose (6.99 \pm 0.77$)$, total cholesterol $(2.20 \pm 0.27)$, triglycerides $(0.58 \pm 0.07)$, TGO $(138.83 \pm 9.16)$, and ALT $(12.47 \pm 2.82$.
\end{abstract}

INDEX TERMS: Wild animals, crocodiles, Melanosuchus niger, Aligatoridae, biochemical profile, serology.

RESUMO.- 0 jacaré-açu (Melanosuchus niger), pertencendo à família Aligatoridae, é considerado o maior predador aquático da América Continental. Como todos os répteis, possui particularidades para o uso de energia, controlando sua temperatura corporal nas variações climáticas. Essas variações podem revelar a situação metabólica atual desse animal, justificando o estudo de tais parâmetros. A importância desse trabalho se justifica pela escassez de estudos disponíveis na literatura. Nesse sentido, determinamos o perfil bioquímico de compostos sanguíneos de 12 jacarés açu, com peso de 77,66 $\pm 8,92 \mathrm{~kg}$, os quais foram: uréia $(3,41 \pm 0,66)$, albumina $(24,85 \pm 0,63)$, proteína total $(63,83 \pm 16,97)$, ácido úrico $(0,25 \pm 0,09)$, glicose $(6,99 \pm 0,77)$, colesterol total $(2,20 \pm 0,27)$, triglicérides $(0,58 \pm 0,07)$, TGO $(138,83 \pm 9,16)$ e TGP $(12,47 \pm 2,82)$.

TERMOS DE INDEXAÇ̃̃O: Animais silvestres, crocodilos, Melanosuchus niger, Aligatoridae, constituintes biquímicos, sorologia.

\footnotetext{
${ }^{1}$ Recebido em 8 de julho de 2015.

Aceito para publicação em 10 de dezembro de 2015.

${ }^{2}$ Laboratório de Ensino e Pesquisa em Animais Silvestres (LAPAS), Universidade Federal de Uberlândia (UFU), Uberlândia, Rua Ceará s/n, Bloco 2D, Sala 36, Bairro Umuarama, Uberlândia, MG 38405-315, Brasil. *Autor para correspondência: brcaixeta@yahoo.com.br
}

\section{INTRODUÇÃO}

Embora o conhecimento sobre a história natural de crocodilianos da América do Sul tem aumentado ao longo das últimas décadas, a conservação deste grupo de animais ainda é dificultada pela escassez de informações sobre sua ecologia básica (Huchzermeyer 2003).

As 6 espécies existentes no Brasil, segundo a Sociedade Brasileira de Herpetologia popularmente conhecidos como jacarés, pertencem à Família Alligatoridae (Villaça 2004).

O gênero Melanosuchus compreende apenas a espécie Melanosuchus niger, conhecido popularmente como jacaré açú ou jacaré preto, considerado o maior predador aquático da América Continental (Thoisay et al. 2006).

De acordo com Andreotti et al. (1995) a espécie Melanosuchus niger, como todos os répteis, possuem particularidades para o uso de energia, controlando sua temperatura corporal principalmente através das variações climáticas em diversos momentos fisiológicos.

Os répteis dependem exclusivamente da temperatura ambiental, pois precisam elevar sua temperatura corporal para diversas atividades homeostáticas.

Fatores como idade, tamanho, sexo, estado nutricional, peso e temperatura do ambiente podem afetar os parâmetros de maneira geral, dificultando o estabelecimento de 
valores séricos de referência para determinadas espécies e posteriores comparações de populações e indivíduos (Franzo \& Vulcani 2010).

O conhecimento das variações fisiológicas dos jacarés são pouco estudadas, bem como sua relação com os valores de parâmetros bioquímicos, e estes são importantes indicadores de normalidade e avaliações posteriores.

Mundim (2008) enfatizou que o perfil bioquímico expressa uma interpretação sobre as condições de saúde do animal que são essenciais para a manutenção de uma série de fatores, dentre eles, o desenvolvimento e capacidade produtiva, dependentes da constância entre funções dos sistemas orgânicos.

De acordo com Batista et al. (2002a) o conhecimento das dosagens bioquímicas em animais domésticos é bem conhecida. Estas mesmas determinações em outros grupos de animais como nos jacarés se fazem necessárias no intuito de contribuir para obtenção de valores de referência para machos, adultos e no entendimento das condições fisiológicas, resultantes de vários fatores, já citados anteriormente, da espécie em questão.

0 estudo se fez devido à escassez de trabalhos disponíveis na literatura, e da importância que representam as variações bioquímicas e fisiológicas dos jacarés, o que limita a comparação dos resultados obtidos neste estudo com trabalhos realizados previamente. Essa limitação fez com que alguns resultados obtidos fossem confrontados com estudos que utilizaram outros répteis.

Segundo Santos et al. (2011) a investigação bioquímica é essencial para buscar valores auxiliares no diagnóstico de possíveis distúrbios patológicos, e posteriormente na avaliação do manejo e adaptação dos mesmos em ambientes de cativeiro aliado com as características climáticas no habitat desses indivíduos, justificando a importância de estudar tais parâmetros e sua relevância para a comunidade acadêmica.

Com esse projeto pretendeu-se aprofundar o conhecimento sobre a fisiologia do animal, quantificar, estudar, caracterizar e divulgar os parâmetros bioquímicos sérico dos jacarés, em específico, do M. niger contribuindo assim para enriquecimento bibliográfico da espécie, pois irá enriquecer os estudos da espécie em questão.

\section{MATERIAL E MÉTODOS}

Foram utilizadas amostras de sangue venoso de 12 jacarés da espécie açú, sendo 12 machos, adultos, classe IV encontrados em habitat de vida livre em riachos, lagos e lagoas de diversas fazendas do município do Estado de Goiás e Mato Grosso, escolhidas previamente pela quantidade de animais, com temperatura ambiente máxima de $33^{\circ} \mathrm{C}$ e mínima de $31^{\circ} \mathrm{C}$, coletadas no mês de outubro de 2014.

As capturas ocorreram no período da noite, decorrente dos hábitos noturnos dos jacarés, preferencialmente entre 19 e $24 \mathrm{~h}$ ou até obter o número suficiente de animais e a localização dos jacarés ocorreu através do reflexo dos olhos quando iluminados com o uso de farol do tipo "sealed beam" ligados à bateria de 12 volts.

Os jacarés foram capturados manualmente com auxílio de um laço, captura tradicionalmente realizada pelos comunitários, onde os jacarés tiveram a boca amarrada com ligas de borracha e fita adesiva $50 \times 50 \mathrm{~mm}$ transparente polipropileno (FITPEL), os olhos fechados com fita adesiva, os membros dianteiros e traseiros levemente amarrados de forma a não provocar edemas (Magnusson \& Canto 2010).

A biometria dos animais capturados foram avaliadas e relacionadas através de modelos matemáticos: $\mathbf{C f c}=$ comprimento focinho/cloaca, em $\mathrm{cm}$ (da ponta do focinho à cloaca) e $\mathbf{P}=$ peso, em quilogramas. Como material tomado para a coleta dos dados biométricos, foi utilizado: uma trena de precisão de $1 \mathrm{~mm}$ para a medida de Cfc, e para variável P por meio de uso de dinamômetro de precisão de marca Pesola de precisão tolerância máxima +/0,3\% de carga (posição 0 exatamente ajustada). A classificação da biometria foi realizada por classes de acordo com Velasco et al. (2009).

A coleta de $2 \mathrm{ml}$ de sangue total se deu por punção do seio venoso cervical com auxílio de seringas de volume total de $5 \mathrm{ml}$, que foram transferidos imediatamente para frascos estéreis (tubos de vidro) de volume total de $5 \mathrm{ml}$ sem anticoagulante, previamente identificados. Logo em seguida os jacarés foram liberados aos arredores do local de captura. Após a coagulação do sangue, as amostras foram centrifugadas a $720 \mathrm{~g}$ por 5 minutos, $1 \mathrm{ml}$ de soro transferido por pipetas semi-automáticas de volume total $1.000 \mu \mathrm{l}$ para tubos plásticos tipo eppendorf e resfriados em caixas isotérmicas contendo gelo. Posteriormente congelados a $-20^{\circ} \mathrm{C}$ em freezer no acampamento mais próximo e transferidos para o Laboratório do Animais Silvestres - LAPAS da Universidade Federal de Uberlândia, localizado na cidade de Uberlândia, MG.

0 soro foi encaminhado em caixas isotérmicas contendo gelo para o Laboratório de Análises Clínicas da Faculdade Patos de Minas, situado na cidade de Patos de Minas, M.G. onde foi processado.

A determinação dos valores bioquímicos foi realizada por meio da análise sorológica quantitativa dos indicadores: albumina pelo método de verde de bromocresol, proteína total pelo método do biureto, ácido úrico pelo método enzimático Trinder, uréia pelo método enzimático cinético Ultra Violeta, glicose pelo método enzimático Trinder, colesterol total pelo método enzimático colorimétrico, triglicérides pelo método enzimático colorimétrico, ALT = alanina aminotransferase e AST = aspartato aminotransferase, ambas pelo método cinético Ultra Violeta. Todas as metodologias descritas anteriormente seguiram a recomendação de cada indicador bioquímico.

As análises foram processadas colorimetricamente em analisador automático - Flexor E, previamente calibrado (Elitech elical II)e aferido com soro controle (Control lab). Foram utilizados Kits comerciais da Elitech Clinical Systems-SAS e Bioclin para dosagem de proteínas totais.

Todos os parâmetros foram submetidos a análise de variância sendo expressos como média e desvio padrão. Em seguida foram determinados os limites inferiores e os limites superiores do intervalo de confiança de 95\% para a média dos indivíduos. Os procedimentos de análises foram feitos na ferramenta Action (2013) (www.portalaction.com.br) que utiliza o programa R (R Development Core Team, 2013). A conversão para o sistema internacional de unidades foi realizada segundo BIOTÉCNICA (2007), visando comparação com a literatura existente. 0 projeto foi submetido e aprovado pela Comissão de Ética na Utilização de Animais (CEUA) sob o número de protocolo 154/13.

\section{RESULTADOS E DICUSSÃO}

Os 12 jacarés capturados para as coletas de sangue apresentaram Cfc acima de $1.80 \mathrm{~m}$, considerados classe IV, portanto adultos. Apresentaram peso de 77,66 $\pm 8,92 \mathrm{~kg}$. Os resultados sorológicos encontrados estão dispostos no Quadro 1. 
Quadro 1. Média e desvio-padrão dos valores de bioquímica sanguínea para jacarés da espécie Melanosuchus niger, expressos em unidades convencionais e no sistema internacional de unidades

\begin{tabular}{lcc}
\hline Variável & Unidade Convencional & $\begin{array}{c}\text { Sistema Internacional } \\
\text { de Unidades }\end{array}$ \\
\hline Albumina & $2,48 \mathrm{~g} / \mathrm{dl}( \pm 0,06)$ & $24,85 \mathrm{~g} / \mathrm{L}( \pm 0,63)$ \\
Ácido Úrico & $4,54 \mathrm{mg} / \mathrm{dl}( \pm 1,55)$ & $0,25 \mathrm{mmol} / \mathrm{L}( \pm 0,09)$ \\
Colesterol & $85,25 \mathrm{mg} / \mathrm{dl}( \pm 10,43)$ & $2,20 \mathrm{mmol} / \mathrm{L}( \pm 0,27)$ \\
Glicose & $126,16 \mathrm{mg} / \mathrm{dl}( \pm 14,01)$ & $6,99 \mathrm{mmol} / \mathrm{L}( \pm 0,77)$ \\
Proteína Total & $6,38 \mathrm{~g} / \mathrm{dl}( \pm 1,69)$ & $63,83 \mathrm{~g} / \mathrm{L}( \pm 16,97)$ \\
TGO/AST & $138,83 \mathrm{UI} / \mathrm{L}( \pm 9,16)$ & $138,83 \mathrm{UI} / \mathrm{L}( \pm 9,16)$ \\
TGP/ALT & $12,47 \mathrm{UI} / \mathrm{L}( \pm 2,82)$ & $12,47 \mathrm{UI} / \mathrm{L}( \pm 2,82)$ \\
Triglicérides & $51,83 \mathrm{mg} / \mathrm{dl}( \pm 4,94)$ & $0,58 \mathrm{mmol} / \mathrm{L}( \pm 0,07)$ \\
Ureia & $3,41 \mathrm{mg} / \mathrm{dl}( \pm 0,66)$ & $0,56 \mathrm{mmol} / \mathrm{L}( \pm 0,10)$
\end{tabular}

As proteínas são importantes para o crescimento e desenvolvimento do organismo, sua principal finalidade nutricional é proporcionar aminoácidos que serão utilizados na síntese de proteínas do próprio organismo para o crescimento dos tecidos do corpo.

A quantificação das proteínas totais determina grosseiramente todas as proteínas presentes na porção de plasma do sangue. Elas formam a base celular, mantém a pressão coloido-osmótica, catalisam reações bioquímicas na forma enzimática, atuam como reguladores hormonais, como anticorpos e mantém equilíbrio ácido-base (Lopes et al. 2007).

De acordo com Campbell (2006) os valores normais de proteína plasmática total geralmente variam de 3 a $7 \mathrm{~g} / \mathrm{dL}$ em répteis. Estudando outras espécies de jacarés, Troiano et al. (1997), observou valores de proteínas totais entre $5,76 \pm 1,24 \mathrm{~g} / \mathrm{dL}$ para C. latirostris e $5,61 \pm 2,45 \mathrm{~g} / \mathrm{dL}$ para $C$. $c$. jacaré; e para albumina entre $2,31 \pm 1,57 \mathrm{~g} / \mathrm{dL}$ e $2,58 \pm 1,35 \mathrm{~g} /$ dL respectivamente, observando que estão próximos aos apresentados, corroborando o presente estudo. Outros autores quantificaram concentrações plasmáticas superiores as médias obtidas, Santos et al. (2010) em C. crocodilus encontrou média de 45,32 $( \pm 12,86) \mathrm{g} / \mathrm{dL}$ e Santos et al. (2010) em Melanosuchus niger de 41,4 $( \pm 11,3) \mathrm{g} / \mathrm{dL}$.

A determinação das proteínas totais pode indicar o estado nutricional e pode ser utilizado para diagnosticar doença renal, doença hepática e outras patologias. Estes valores baixos podem ser encontrados em situações de desnutrição grave ou em condições que causam má-absorção. Níveis elevados de proteínas podem ocorrer em situações de inflamação crônica, infecciosas e de doenças da medula óssea (Santos 1997, Lopes et al. 2007).

No presente estudo os níveis encontrados para globulinas foram de $3,90( \pm 1,63) \mathrm{g} / \mathrm{dL}$. A concentração de globulinas é obtida quando é realizada a diferença entre proteínas totais e albumina. A elevação dos níveis de globulina inibe a síntese de albumina no fígado como forma de contrapartida para manter o nível proteico total constante e assim a pressão osmótica sanguínea. Ao contrário quando existe uma disfunção hepática a queda nos níveis de albumina, mas elevação das globulinas (Contreras 2000).

Comparando valores encontrados por Troiano et. al. (1995) em outras espécies de répteis, como Eunectes notaeus, foram observados valores superiores aos encontra- dos no presente trabalho para proteínas totais e valores inferiores para albumina e ácido úrico.

Essas variações encontradas podem ser decorrentes da atividade predatória dos jacarés, pois consumem proteína animal quase que exclusivamente para sobrevivência, manutenção e crescimento. Se o teor calórico de aminoácidos ingeridos exceder o requerimento para a síntese proteica e para a energia, o corpo destina esses aminoácidos ao tecido adiposo.

0 ácido úrico é o produto final do metabolismo de proteínas em répteis e importante no que diz respeito ao balanço hídrico dos mesmos. 0 nitrogênio dos aminoácidos não usados, passam para amônia nos rins e para ácido úrico no fígado. Santos (1997) ressalta ainda que os animais não podem sintetizar efetivamente as proteínas, quando a temperatura está abaixo de $20^{\circ} \mathrm{C}$, devido à baixa taxa metabólica dos animais. No presente trabalho os valores médios para a dosagem de ácido úrico foram $0,25 \pm 0,09 \mathrm{mmol} / \mathrm{L}$, porém não foram encontrados estudos com dosagens para o mesmo analito em outras espécies de jacarés.

A hiperuricemia pode ser prejudicial quando depositado nas articulações, o que implica em grande parte na dificuldade de locomoção dos animais, podendo ocorrer paralisia e distendimento dos membros, principalmente dos dedos. É um bom indicador em casos de desidratação e podem também indicar insuficiência renal (nos casos de deposição nos rins) e estão correlacionados com diversas condições ou até ocasionar a morte (Santos 1997).

Já para Troiano et al. (1997), os valores séricos de uréia $(7,75 \pm 2,61 \mathrm{mmol} / \mathrm{L})$ e $(10,20 \pm 1,24 \mathrm{mmol} / \mathrm{L})$ em espécies de Caiman latirostris e Caiman c. jacaré respectivamente, encontravam bem superiores aos que o presente estudo relata, o que não foi observado por Santos et al. (2010), com valores de uréia $(1,2 \pm 0,4 \mathrm{mmol} / \mathrm{L})$. No entanto, em répteis, o nível de creatinina encontrado geralmente é baixo e não parece ser um bom indicador de doença renal (Campbell 2006). Segundo Almosny \& Monteiro (2007), a relação uréia/creatinina em répteis pode ser usada como indicador de catabolismo proteico e de doença renal. Apesar desse trabalho não ter quantificado a creatinina para comparações, essa abordagem ainda carece de padronização para a espécie.

As variações observadas podem ser provenientes de patologias renais e pré-renais que estejam acometendo alguns indivíduos, visto que, as coletas foram realizadas em animais livres aparentemente normais.

Os valores publicados sobre estudos relacionados à bioquímica sérica dos crocodilianos, grande parte ainda é desconhecida e variam de espécie para espécie. Assim como os obtidos de animais de vida livre, uma vez, que estes estão expostos a inúmeras variáveis.

No entanto, eles podem demonstrar uma grande orientação para a interpretação dos resultados de investigações clínicas.

Para o caso particular do triglicérides e do colesterol total, os valores encontrados nesse estudo são inferiores a todos os valores descritos em outras espécies de jacarés e em até em outros répteis (Troiano et al. 1995, Troiano et al. 1997, Troiano et al. 1998, Santos et al. 2010).

Essas variações podem ser provenientes da diversidade e abundância alimentar decorrentes das diferentes regiões 
de habitat, alterações das estações climáticas, como a seca e a época das cheias que, por conseguinte pode influenciar no tipo de alimento capturado e ingerido pelos jacarés e de certa forma intervir nos níveis dos marcadores bioquímicos.

Os componentes bioquímicos sanguíneos determinados em um perfil metabólico representam as principais vias da taxa metabólica do organismo. Essa interpretação é complexa, devido aos mecanismos que controlam o nível sanguíneo de vários metabólitos e a interferência dos fatores próprios de cada indivíduo, causando grande amplitude desses níveis (Mundim 2008).

Não obstante às proteínas e os lipídeos, a taxa glicêmica representa um importante indicador orgânico. Para Batista et al. (2002b), a concentração dos níveis de glicose sanguínea dos répteis varia sazonalmente de acordo com a espécie, condições bióticas e estado nutricional. Ainda de acordo com o estudo, nos alligators, no inverno, época semelhante ao período seco no pantanal(região onde ocorreram as coletas) ocorre uma diminuição do apetite, ocasionando uma diminuição do nível de glicose.

Os valores de glicose plasmática encontrados neste estudo foram de $6,99 \mathrm{mmol} / \mathrm{L}( \pm 0,77)$ e estão próximos dos descritos por Santos et al. (2010) com Melanosuchus niger $5,1( \pm 0,9) \mathrm{mmol} / \mathrm{L}$. Troiano et al. (1997) relataram valores superiores aos descritos para $C$. crocodilus nas espécies C. latirostris $98,80( \pm 17,09) \mathrm{mmol} / \mathrm{L}$ e C. c. yacare 77,80 $( \pm 10,76) \mathrm{mmol} / \mathrm{L}$.

As diferenças entre os valores encontrados no presente trabalho com os demais autores podem ser atribuídas a fatores como idade, tipo de alimentação, condições ambientais, além de metodologia, reagentes e analisadores bioquímicos nos processamentos das análises.

Os resultados encontrados neste estudo para as dosagens de AST e ALT estão muito próximos dos valores descritos por Troiano et al. (1997), em análises realizadas em espécies de C. c. jacaré $(138,5 \pm 21,30 \mathrm{UI} / \mathrm{L} ; 18,0 \pm 10,55 \mathrm{UI} / \mathrm{L})$ e Caiman latirostris $(135,5 \pm 22,49 \mathrm{UI} / \mathrm{L} ; 14,26 \pm 5,86 \mathrm{UI} / \mathrm{L})$ respectivamente. Comparando com valores encontrados em Troiano et. al. 1998, valores para AST encontraram-se menores e próximos para ALT, em espécies de B. alternatus mantidas em cativeiro.

As diferenças ocorridas entre os níveis dos constituintes bioquímicos do sangue de Melanosuchus niger deste estudo, quando comparados com os dados de outros pesquisadores se devem, provavelmente, às diferentes espécies, sexo, habitats, alimentação e mesmo a variação de idade, pois são animais, que possuem uma vida longa. Podem ser que algumas destas variações sejam em decorrência das variações fisiológicas, temperatura e estresse durante a manipulação dos animais. Poucos fatores ambientais têm tanta influência sobre a fisiologia do animal como a temperatura. A temperatura corporal influencia diretamente os parâmetros fisiológicos e bioquímicos (Randall 2000, Branco 2005).

\section{CONCLUSÕES}

A utilização de metodologia analítica e de reagentes consagrados para exames bioquímicos em animais domés- ticos se mostrou adequada para determinação dos parâmetros analisados em jacarés.

Os resultados do presente estudo visa contribuir para a obtenção dos valores dos constituintes bioquímicos para machos, adultos da espécie em questão.

Os dados devem ser interpretados com cautela em virtude das inúmeras variáveis encontradas, sugerindo continuidade em novos estudos a fim de entender mais sobre a espécie.

\section{REFERÊNCIAS}

Almosny N.R.P. \& Monteiro A.M. 2007. Patologia clínica, p.939-36. In: Cubas Z.S., Silva J.C.R. \& Dias J.L.C. (Eds), Tratado de Animais Selvagens: medicina veterinária. Roca, São Paulo.

Andreotti R. \& Silva R.A.M.S. 1995. Valores bioquímicos e hematológicos do Caiman crocodilus yacare (Daudin, 1802) na natureza. Revista Científica, UFMS, Corumbá, 2(1):33-38.

Batista K.M., Porfírio L.C. \& Machado C.H. 2002a. Colesterolemia de jacarédo-pantanal (Caiman crocodylus yacare) de 2 anos de idade, criados em cativeiro com alimentação exclusiva de carne bovina e submetidos a jejuns alimentares de 12 a 60 horas. Revta Cient. Cent. Univ. Barra Mansa 4(7):4-6.

Batista K.M., Porfírio L.C., Machado C.H., Marcelino D.P., Meyer Júnior J.C. \& Corrêa J.A. 2002b. Glicemia de Caiman crocodylus yacare criados em cativeiro e submetidos a jejum alimentar de 60 horas. Revta Bras. Med. Vet., Barra Mansa, 24(1):9-11.

Biotécnica 2007. Biotécnica-Tabela de Conversão de Unidades. Disponível em:<http://www.blogbiotecnica.ind.br/blog/2011/04/tabela-de-conversao-conversao-de-unidades-e-diluicao-de-urina-normal>. Acesso em 09 de setembro de 2014.

Branco L.G.S. 2005. Regulação endócrina da temperatura corporal, p.6480. In: Antunes-Rodrigues J., Moreira A.C., Elias L.L.K. \& Castro M. (Eds), Neuroendocrinologia básica e aplicada. Guanabara Koogan, São Paulo.

Campbell T.W. 2006. Clinical pathology of reptiles, p.490-532. In: Mader D.R. (Ed.), Reptile Medicine and Surgery. Saunders Elsevier, Missouri.

Contreras P. 2000. Indicadores do mestabolismo proteico utilizados nos perfis metabólicos de rebanhos, p.23-30. In: Gonzáles F.H.D. (Ed.), Perfil Metabólico em Ruminantes: seu uso em nutrição e doenças nutricionais. Gráfica da UFRGS, Porto Alegre.

Franzo V.S. \& Vulcani V.A.S. 2010. Estudo da fórmula vertebral do jacaréaçú (Melanosuchus niger). Revta Cient. Eletron. Med. Vet., Garça/SP, $6(14)$.

Huchzermeyer F.W. 2003. Crocodiles: biology, husbandry and diseases. CABI Publishing, South Africa. 352p.

Lopes S.T.A., Biondo A.W. \& Dos Santos A.P. 2007. Manual de Patologia Clínica Veterinária. 3a ed. Departamento de Clínica de Pequenos Animais, UFSM, Santa Maria.

Magnusson W.E., Canto S.L.O., Marioni B. \& Thorbjarnarson J. 2010. Feeding behavior of two sympatric caiman species, Melanosuchus niger and Caiman crocodilus, in the Brazilian Amazon. J. Herpetol., Washington, 42(4):768-772.

Mundim A.C. 2008. Perfil bioquímico sérico em potros bretão postier e cães doberman em fase de crescimento e de cabras saanen nos diferentes estágios de lactação. Dissertação de Doutorado em Genética e Bioquímica, Instituto de Genética e Bioquímica, Universidade Federal de Uberlândia, Uberlândia, MG. 88p.

Randall D. 2000. Eckert's Fisiologia Animal. 4ํㅡㄹ ed. Guanabara Koogan, Rio de Janeiro.

Santos M.Q.C., Oliveira A.T., Lemos J.R.G., Silva M.G., Dias M.T. \& Marcon J.L. 2010. Características hematológicas do jacaré-açú Melanosuchus niger Spix, 1825 (Crocodylia, Alligatoridae) da Bacia do Rio Negro, Amazonas, Brasil. 62aㅡ Reunião Anual da SBPC, Natal.

R Development Core Team, 2013. European Evironment Agency. A language and environment for statistical computing. R Foundation for Statistical Computing. Disponível em: <https://www.r-project.org>. Acesso em 14 de novembro de 2014. 
Santos A.L.Q., Mundim A.V., Menezes L.T., Kaminishi A.P.S., Leonardo T.G. \& Nascimento L.R. 2011. Proteinograma sérico de Tartarugas-da-Amazônia (Podocnemis expansa Schweigger, 1812) Testudines, Podocnemididae. Pubvet, Londrina, 5(16), Ed.163, Art.1104.

Santos S.A. 1997. Dieta e Nutrição de Crocodilianos. Embrapa Pantanal, Corumbá. 59p.

Thoisay B.D.E., Hrbek T., Farias I.P., Vasconcelos W.R. \& Lavergne A. 2006. Genetic structure, population dynamics, and conservation of black caiman (Melanosuchus niger). Biological Conservation 1(33):474-482.

Troiano J.C., Althaus R.L., Malinskas G. \& Juan R. 1995. Valores de los principales parâmetros de la bioquímica sanguínea de Eunectes notaeus (Ophidia, Boidae). Facena11:51-55.

Troiano J.C., Althaus R.L. \& Malinskas G. 1997. Perfil bioquímico sanguíneo de las especies argentinas del género Caiman en condiciones de cautividad. Revta Espanhola Herpetol. 11:31-34.

Troiano J.C., Gold E., Malinskas G., Vidal J.C., Scaglione M.C., Gold J., Dinápoli H., Scaglione L.M. \& Roodt A. 1998. Valores de bioquímica sanguíena de Brothrops alternatus (Ophidia, Crotalidae) em cautividad. Revta Espanhola Herpetol. 12:45-48.

Velasco A., Colomine G., Sola R. \& Villarroel G. 2009. Effects of sustained harvests on wild populations of Caiman crocodilus crocodilus in Venezuela. Interciencia, Caracas, 28(9):544-548.

Villaça A.M. 2004. Uso de habitat por Caiman crocodilus e Paleosuchus palpebrosus no reservatório da UHE de Lajeado, Tocantins. Dissertação de Mestrado em Ecologia de Agroecossistemas, Escola Superior de Agricultura "Luiz de Queiros", Universidade de São Paulo, Piracicaba, SP. 59 p. 